\title{
Tracheobronchopathia osteochondroplastica presenting as an isolated nodule in the right upper lobe bronchus with upper lobe collapse
}

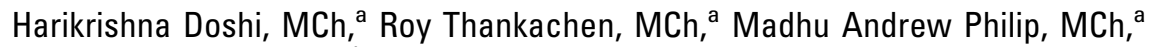

Susy Kurien, MD (Path), ${ }^{\text {b }}$ Vinayak Shukla, MCh, DipNB (CVTS), ${ }^{a}$ and

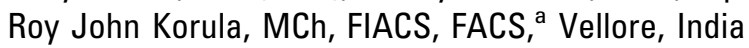

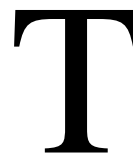

racheobronchopathia osteochondroplastica is a rare benign condition that is characterized by the presence of bony and cartilaginous nodules in the tracheal and bronchial mucosa. (1856), and Wilks (1857). The cause of the disease still remains unknown. Most cases are asymptomatic and frequently diagnosed incidentally during routine bronchoscopies and occasionally during difficult intubations or radiologic examinations.

\section{Clinical Summary}

A 55-year-old woman presented with a history of dry cough and left-sided chest pain associated with low-grade fever of 6 months'
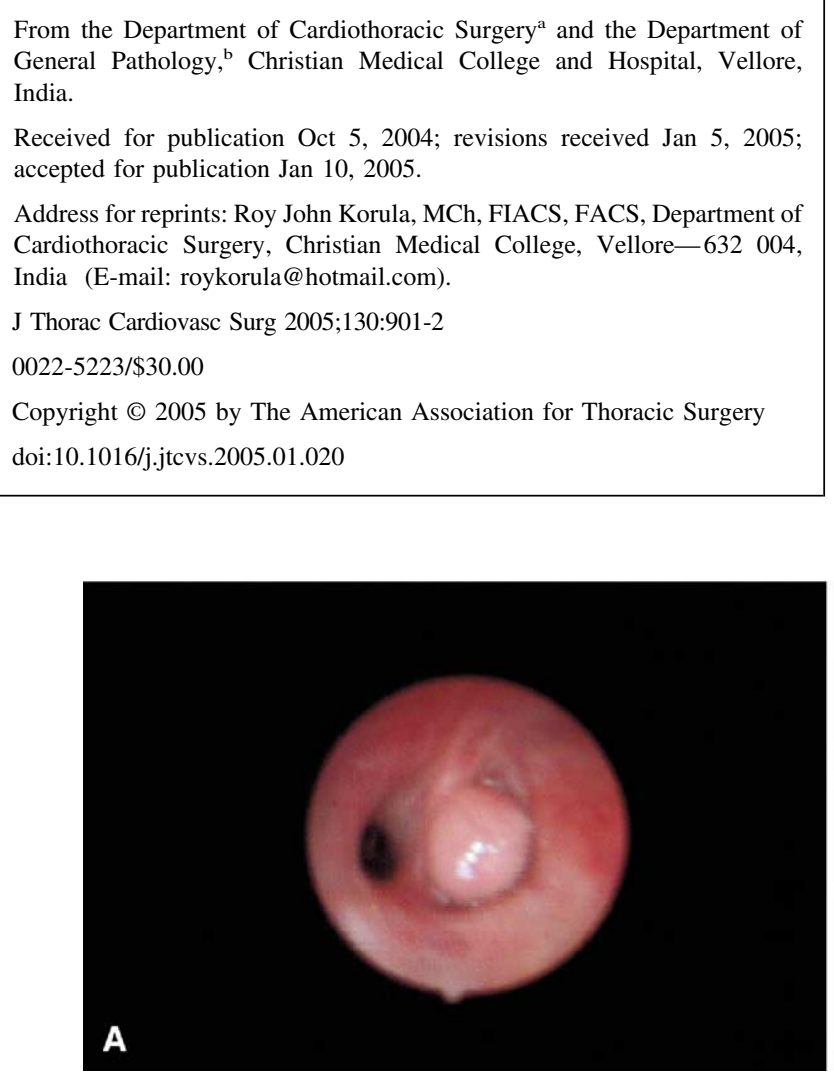

duration. She had been given a diagnosis of tuberculous cervical lymphadenitis 3 years previously and

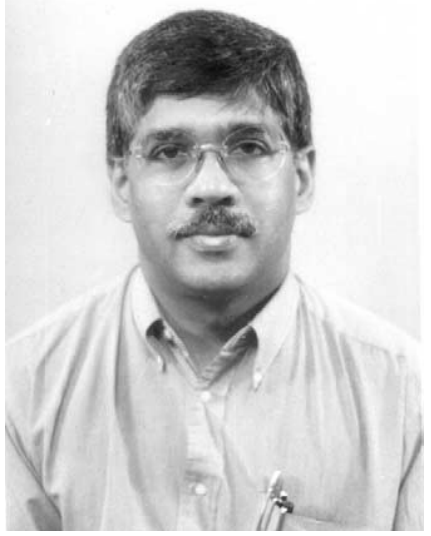

Dr Korula had received a course of antituberculous therapy. The general physical and systemic examination did not reveal any significant abnormality. The chest x-ray film showed no abnormalities. The laboratory profile, including sputum for acid-fast bacilli, was negative. Fiberoptic bronchoscopy showed normal tracheal mucosa with a sharp and mobile carina. The right upper lobe bronchus was completely occluded by a hard mass that was difficult to biopsy. The bronchoscopic biopsy report was inconclusive, and the cytology report was negative for malignancy. A computed tomographic scan of the thorax showed occlusion of the right upper lobe bronchus by a calcified mass with no parenchymal extension and collapse of the lung distally (Figure 1). A right upper lobectomy was performed. There was an intraluminal tumor occluding the right upper lobe bronchus with an adequate stump available for closure after resection. The gross specimen revealed a hard, polypoid, whitish nodule measuring $1.0 \mathrm{~cm}$ in diameter that occluded the right upper lobe bronchus. The lung parenchyma appeared unremarkable. On microscopic examination, the bronchial wall at the site of the nodule showed a polypoidal lesion covered by bronchial epithelium and composed of cartilage and

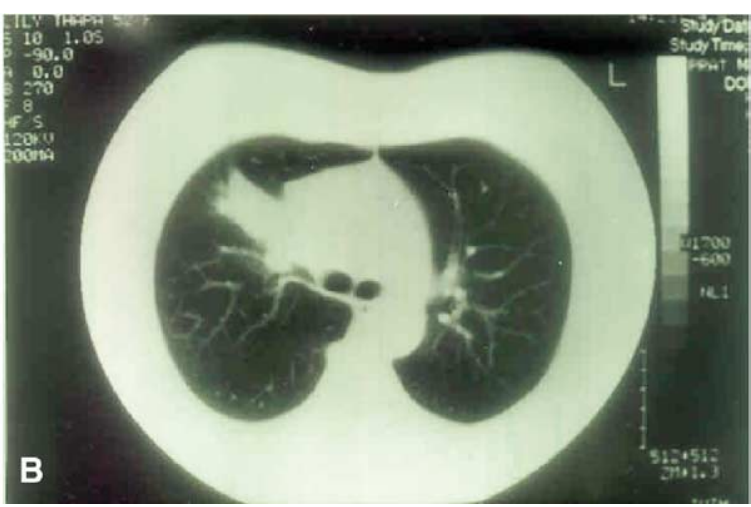

Figure 1. A, Bronchoscopy showing occlusion of the right upper lobe bronchus by the tumor. B, Computed tomographic scan of the thorax showing occlusion of the right upper lobe bronchus by the tumor with collapse of the right upper lobe. 


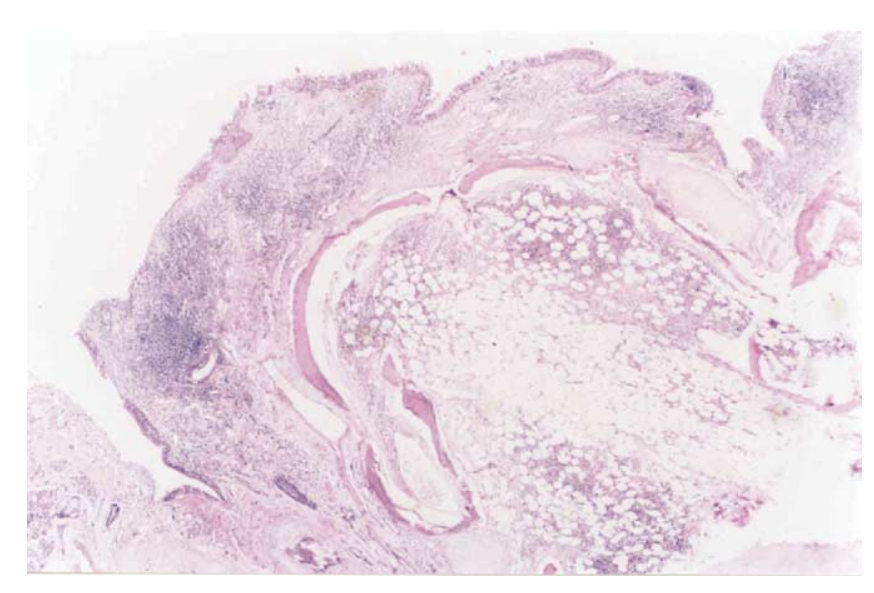

Figure 2. Microscopy showing a polypoidal elevation of the bronchial mucosa composed of cartilage and cancellus bone with marrow. (Hematoxylin and eosin; original magnification $40 \times$.)

cancellus bone with intervening bone marrow, which was suggestive of tracheobronchopathia osteochondroplastica (Figure 2). The adjacent lung showed chronic bronchitis and no evidence of bronchiectasis. The patient had an uneventful recovery.

\section{Discussion}

Tracheobronchopathia osteochondroplastica has been described as a rare benign condition affecting the tracheobronchial tree. The majority of patients with this disorder remain asymptomatic throughout their lives unless airway obstruction develops, in which case they might present with cough, expectoration, and recurrent lower respiratory tract infections. ${ }^{1}$

Chest radiography has been found to be nondiagnostic for the primary lesion. However, in symptomatic patients with airway occlusion, there might be evidence of collapse or bronchiectasis distal to the narrowing in the lumen. The characteristic computed tomographic scan finding of a "beaded or scalloped" appearance of the tracheal wall has not often been documented; however, it can help identify the calcific nodules that often protrude into the lumen. ${ }^{2}$ Bronchoscopic findings have described the lesion as resembling a rock garden or having a cobblestone appearance. ${ }^{3}$

The lesion has most often been reported to occur in the lower two thirds of the trachea and occasionally has been found to involve the proximal bronchi as well. It is rare that exclusive bronchial involvement, as seen in our patient, has been reported. ${ }^{2}$

The cause remains unknown, although there are numerous theories. Chronic infection, congenital anomaly, chemical or mechanical irritation, degenerative or metabolic abnormalities, and genetic predisposition have been considered as possible factors. ${ }^{4}$ The uncertainty of the pathogenesis is evident from the numerous and sometimes contradictory theories that have been proposed. ${ }^{5}$

Historically, tracheobronchopathia osteochondroplastica has not been frequently recognized ante mortem because most patients remain asymptomatic. The treatment is generally conservative in patients with mild symptoms. However, in patients with more debilitating symptoms related to severe airway obstruction, various modalities of treatment, such as endoscopic excision of tumor, laser therapy, and sleeve resections of the trachea have been attempted with varying degrees of success. ${ }^{2}$ Surgical resection of the lung would be indicated in patients with parenchymal lung disease resulting from long-standing occlusion of the airway.

\section{References}

1. Hodges MK, Israel E. Tracheobronchopathia osteochondroplastica presenting as right middle lobe collapse. Chest. 1988;94:842-4.

2. Nienhuis DM, Prakash UBS, Edell ES. Tracheobronchopathia osteochondroplastica. Ann Otol Rhinol Laryngol. 1990;99:689-94.

3. Clee MD, Anderson JM, Johnston RN. Clinical aspects of tracheobronchopathia osteochondroplastica. Br J Dis Chest. 1983;77:308-14.

4. Akyol MU, Martin AA, Dhurandhar N, Miller RH. Tracheobronchopathia osteochondroplastica: a case report and a review of the literature. Ear Nose Throat J. 1993;72:347-50.

5. Meyer N, Dossing M, Broholm H. Tracheobronchopathia osteochondroplastica. Respir Med. 1997;91:499-502. 